\title{
Organizational aspects of global therapy: outcomes
}

\section{Diego Ettore Liberati*}

Dipartimento di Elettronica, Research Director National Research Council of Italy, Milano, Italy

The patient mentioned in my previous letter died after a few months. At the end, she did not suffer too much, and was quite conscious and herself almost till the very last days.

In the last hours, a compassionate morfine and oxygen palliative support helped her to die quietly.

She did not undergo surgery: while the oncologist and the anaesthesist were in favor, the surgeon was afraid of the too advanced stage of the cancer in the left breast, probably implying in his experience a too great difficulty in healing after the cut.
The family had an important involvement especially in term of devoted time, not just to assist the patient, but also to drive her to visits and therapies. In the last days, as said, palliative oxygen and morphine therapy has been provided at home.

It has been a sad and hard experience, as always with cancer death, but in summary one could not really say things have not been well managed.

Is is probably possible to do better, especially when the family has no possibility to be so supportive, but at the end she had the time to prepare herself to die in a not too painful way: thanks God and the therapists
Copyright: (C2018 Liberati DE. This is an open-access article distributed under the terms of the Creative Commons Attribution License, which permits unrestricted use, distribution, and reproduction in any medium, provided the original author and source are credited.
${ }^{*}$ Correspondence to: Diego Ettore Liberati, $\mathrm{PhD}$, Research Director National Research Council of Italy, Information \& Control 4 Systems Biology @ IEIIT, Dipartimento di Elettronica, Informazione e Bioingegneria, Politecnico Piazza Leonardo da Vinci 32, Milano, Italy, E-mail: diego.liberati@polimi.it

Received: June 25, 2018; Accepted: June 28, 2018; Published: June 30, 2018 